\title{
Type-II band alignment of zinc-blende and wurtzite segments in GaAs nanowires: A combined photoluminescence and resonant Raman scattering study
}

\author{
Patryk Kusch, ${ }^{1, *}$ Eugen Grelich, ${ }^{1}$ Claudio Somaschini,${ }^{2}$ Esperanza Luna, ${ }^{2}$ Manfred Ramsteiner, ${ }^{2}$ Lutz Geelhaar, ${ }^{2}$ \\ Henning Riechert, ${ }^{2}$ and Stephanie Reich ${ }^{1}$ \\ ${ }^{1}$ Institut für Experimentalphysik, Freie Universität, Berlin 14195, Germany \\ ${ }^{2}$ Paul-Drude-Institut für Festkörperelektronik, Berlin 10117, Germany
}

(Received 10 October 2013; revised manuscript received 13 January 2014; published 27 January 2014)

\begin{abstract}
We used spatially resolved photoluminescence (PL) and resonant Raman spectroscopy to study the electronic structure of single GaAs nanowires (NWs) consisting of zinc-blende (ZB) and wurtzite (WZ) segments. For narrow ZB segments and stacking faults the energy range of the observed PL peak positions is found to deviate from that of the maxima in resonance Raman profiles. These different energy ranges reflect the fact that the PL recombination is dominated by spatially indirect transitions whereas the resonance enhancement of Raman scattering is caused by direct transitions. Our results provide evidence for the type II band alignment between $\mathrm{ZB}$ and WZ GaAs and a coherent picture of all near-band-gap transition energies in GaAs NWs.
\end{abstract}

DOI: 10.1103/PhysRevB.89.045310

PACS number(s): 78.30.Fs, 63.20.kd

GaAs nanowires (NWs) are fascinating quasi-onedimensional crystals with a huge potential for application in the field of optoelectronics, e.g., as light-emitting diodes, photodetectors, and other devices [1-7]. In its bulk form, GaAs crystallizes in the ZB structure. Due to the lower surface energy of the WZ phase and the large surface-to-volume ratio of nanostructures, GaAs NWs may show a pure $\mathrm{WZ}$, a pure $\mathrm{ZB}$, or a mixture of $\mathrm{ZB}$ and $\mathrm{WZ}$ phases [8-10].

Despite the strong interest in the WZ phase of GaAs, its electronic properties are controversially discussed in the literature, in particular the fundamental electronic band-gap energy (reported values between 1.37 and $1.48 \mathrm{eV}$ ) as well as the nature of the lowest conduction band minimum [11-25]. Recently, even different temperature dependence of the band gaps in ZB and WZ GaAs were found, resulting in similar band-gap energies at low temperature and a larger band gap for $\mathrm{WZ} \mathrm{GaAs}$ at room temperature [26]. In many cases, the band-gap energies were extracted from low-temperature luminescence spectra of GaAs NWs. The dominant PL peaks of WZ GaAs were sometimes observed lower and sometimes higher in energy than the ZB band gap. A plausible explanation for this variation is a type II band alignment in GaAs NWs consisting of $\mathrm{ZB}$ and $\mathrm{WZ}$ GaAs segments[27,28]. In the corresponding model, the relevant electronic properties of stacking faults (SFs) and narrow ZB insertions surrounded by wider WZ GaAs segments are both approximated by a quantum-well formation in the conduction band only, as discussed by Spirkoska et al. [17] and Jahn et al. [22]. In luminescence experiments, the large carrier diffusion length in GaAs (on the order of $1 \mu \mathrm{m}$ ) leads to the capture of electrons within SFs or ZB insertions followed by their recombination via spatially indirect transitions with holes in the valence band of adjacent WZ material. As a consequence the energies of the effective direct band gaps in such quantum-well structures cannot be directly observed in luminescence experiments. Furthermore, luminescence measurements alone [17,22] are not sufficient to rigorously prove the type II band alignment between $\mathrm{ZB}$ and WZ GaAs.

\footnotetext{
*patryk.kusch@fu-berlin.de
}

In this paper we report PL and resonant Raman experiments on single GaAs NWs containing ZB and WZ segments as well as SFs in wider WZ segments. Similar to optical absorption and in contrast to PL experiments, resonant Raman profiles are dominated by spatially direct transitions [29]. We demonstrate that the combination of position-dependent PL and resonant Raman spectroscopy provides an experimental proof for the type II band alignment in ZB/WZ GaAs heterostructures.

GaAs NWs were grown by molecular beam epitaxy (MBE) directly on a $\mathrm{Si}$ (111) substrate, through the Au-assisted vapor-liquid-solid (VLS) mechanism. An oxide-free Si (111) wafer was loaded into the MBE just after an HF dip, which ensures the absence of $\mathrm{a} \mathrm{SiO}_{x}$ layer on the substrate surface, and annealed at around $630^{\circ} \mathrm{C}$ for $20 \mathrm{~min}$ before the $\mathrm{Au}$ deposition. A 0.6-nm-thick layer of $\mathrm{Au}$ was deposited in the growth chamber at $500{ }^{\circ} \mathrm{C}$ and annealed for 10 min under As flux at the same temperature. The NW growth was then initiated by opening the Ga shutter, with a beam flux equivalent to $400 \mathrm{~nm} / \mathrm{h}$ for planar GaAs growth on GaAs (001) substrates, a V/III ratio of 2 and a total growth time of $2 \mathrm{~h}$. Under similar growth conditions high-quality GaAs NWs with predominant WZ crystal phases have already been demonstrated [22,30,31]. For the first hour of NW growth, the substrate temperature was kept at $500^{\circ} \mathrm{C}$, then it was ramped down to $360^{\circ} \mathrm{C}$ without any growth interruption. Growing at lower temperatures increased the radial growth rate and resulted in an enlargement of the NW diameter, which facilitates the spectroscopic investigations on single, dispersed NWs.

The resulting NWs have an average diameter of around $100 \mathrm{~nm}$ at the bottom and around $200 \mathrm{~nm}$ in the middle part, are pencil-shaped at their very top, and exhibit a length of $10 \mu \mathrm{m}$. However, the sizes and shapes of the NWs were found to vary; mainly, we observed a minimum of $120 \mathrm{~nm}$ and a maximum of $220 \mathrm{~nm}$ for the diameter in the middle part of the wire, but some wires also showed diameters below $50 \mathrm{~nm}$. Figure 1(a) shows an scanning-electron microscopy (SEM) image of as-grown NWs.

For the combined PL and Raman experiments, the NWs were removed from the growth substrate by ultrasonication in ethanol and dispersed on a $\mathrm{Si}$ substrate with markers for identification under a light microscope. The solution 


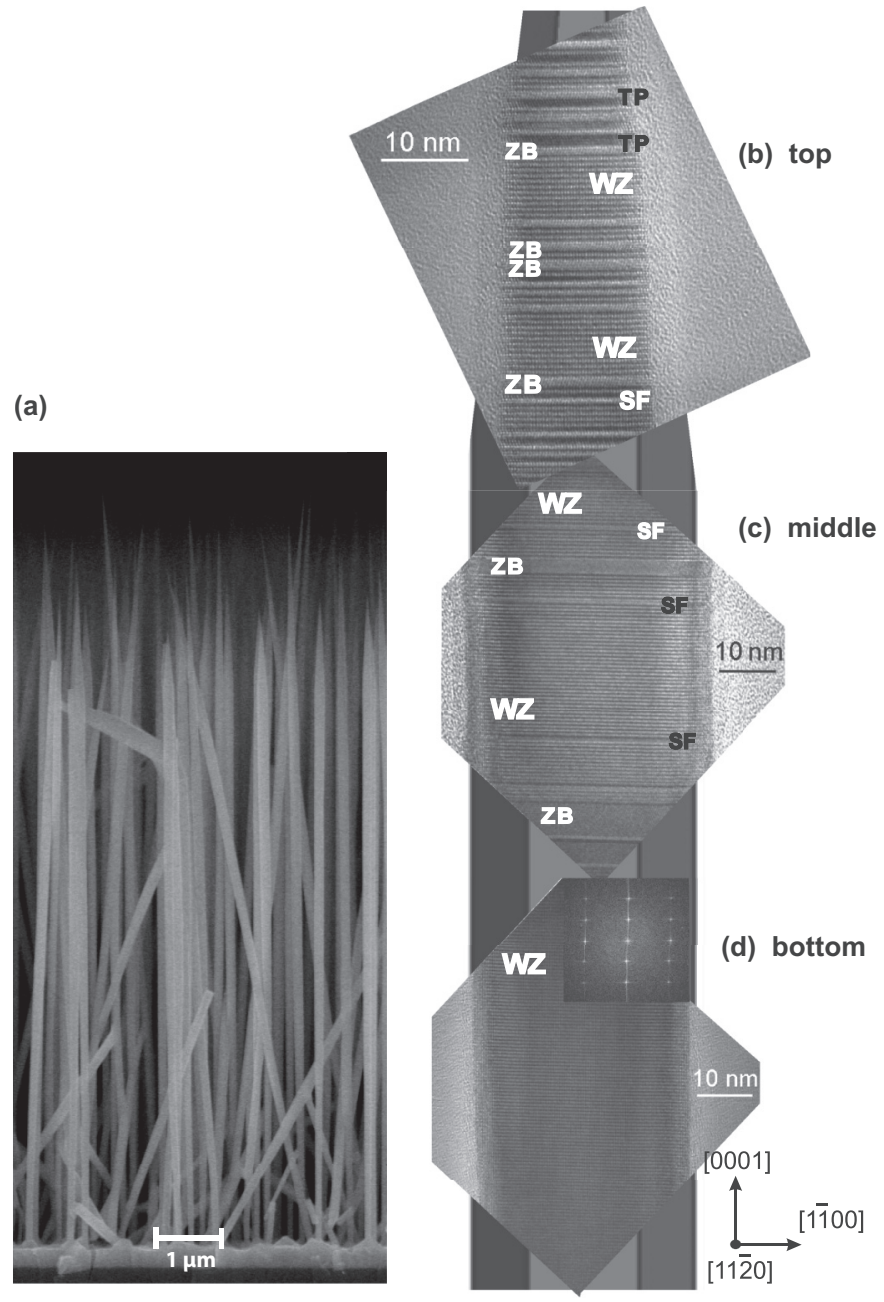

FIG. 1. (a) SEM micrograph of NWs used for PL and resonant Raman experiments. TEM images of the top (b), middle (c) and bottom (d) part of a single GaAs NWs. ZB marks zinc-blende segments, SF stacking faults, TP twin planes, WZ marks the predominant wurtzite crystal structure. Similar morphologies were observed for other wires.

was diluted to achieve single-nanowire measurements. In atomic force microscopy (AFM) measurements the NW, which was used for the resonant Raman and PL experiments, was observed to have a diameter of $110 \mathrm{~nm}$ at the bottom, $190 \mathrm{~nm}$ at the middle, and $80 \mathrm{~nm}$ at the top. A Ti:sapphire ring laser was used for optical excitation in the wavelength range from 730 to $890 \mathrm{~nm}(1.39-1.69 \mathrm{eV})$ with a constant power of $3.5 \mathrm{~mW}$. The spectra were recorded with a Horiba T64000 triple monochromator in backscattering configuration at room temperature. The laser beam was focused on the selected NW by a microscope objective which was used for the collection of the PL/Raman light as well. Due to the band symmetry of wurtzite $\mathrm{GaAs}$ ( $\Gamma_{9}$ conduction and $\Gamma_{7}$ valance band) $[12,31,32]$, the incoming and outgoing light was chosen to be polarized perpendicular to the $z$ axis $[x(y, y) \bar{x}$ configuration], where $z$ is the growth axis $c$ [33]. The position of excitation along the NW axis was controlled by a motorized $X Y$ stage. The Raman peaks were fitted by Lorentzians and the intensities were determined by peak area integration. The intensity was calibrated using a $\mathrm{CaF}_{2}$ crystal with a known and constant Raman cross section [29]. Note that the measured PL peaks are dominated by band-to-band recombination since shallow defects are ionized at room temperature $(297 \mathrm{~K})$.

To determine the microstructure of the GaAs NWs, transmission electron microscopy (TEM) was performed on a large number of individual wires. All investigated NWs exhibited similar characteristics. Representative TEM micrographs of a single GaAs NW with a diameter of $40 \mathrm{~nm}$ are shown in Fig. 1. At the bottom [Fig. 1(d)] the GaAs NWs have wurtzite structure with no or very few isolated SFs. In the middle part of the NW, the predominant crystal structure is wurtzite, whereas the density of isolated SFs increases considerably with respect to that at the bottom [cf. Fig. 1(c)]. Furthermore, $\mathrm{ZB}$ insertions (marked as ZB in the figure) are also detected. These consist of nanometer-sized thin segments of $\mathrm{ZB} \mathrm{GaAs}$ material. Finally, at the top of the NW there are still signatures of the predominant WZ structure [Fig. 1(b)]. We observe a high density of ZB insertions, the total amount of ZB segments is much larger than in the middle part. Additionally, some ZB $\mathrm{GaAs}$ areas are defective and exhibit twinning. The twin planes (TP) are marked in the figure. The extent and the defective condition of the ZB segments at the top part of the NW vary slightly from NW to NW. Due to the different microstructure at the top, middle, and bottom part of the NW and taking advantage of the high spatial resolution of the resonant Raman scattering technique (about $0.7 \mu \mathrm{m}$ ), we further analyzed the properties of all three parts of the NW separately.

Combined Raman and PL spectra are shown in Fig. 2, recorded at different positions along the axis of a single NW. Because of the excitation at a photon energy only $160 \mathrm{meV}$ above the band gap of $\mathrm{ZB} \mathrm{GaAs,} \mathrm{it} \mathrm{is} \mathrm{possible} \mathrm{to} \mathrm{observe}$ PL and Raman scattering simultaneously. First-order Raman scattering by transversal optical (TO) and longitudinal optical (LO) phonon modes is observed at 267 and $293 \mathrm{~cm}^{-1}$, respectively. In addition resonant second-order Raman scattering by

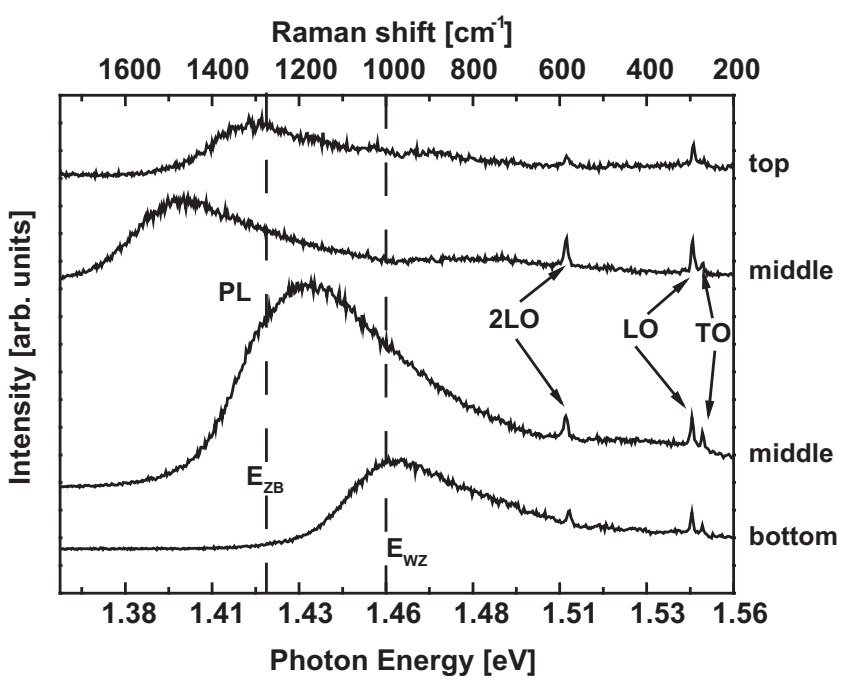

FIG. 2. Combined PL and Raman spectra recorded at different positions along the growth axis of a GaAs NW for excitation at a photon energy of $1.583 \mathrm{eV}$. The vertical dashed lines indicate the band-gap energies of ZB and WZ GaAs. 


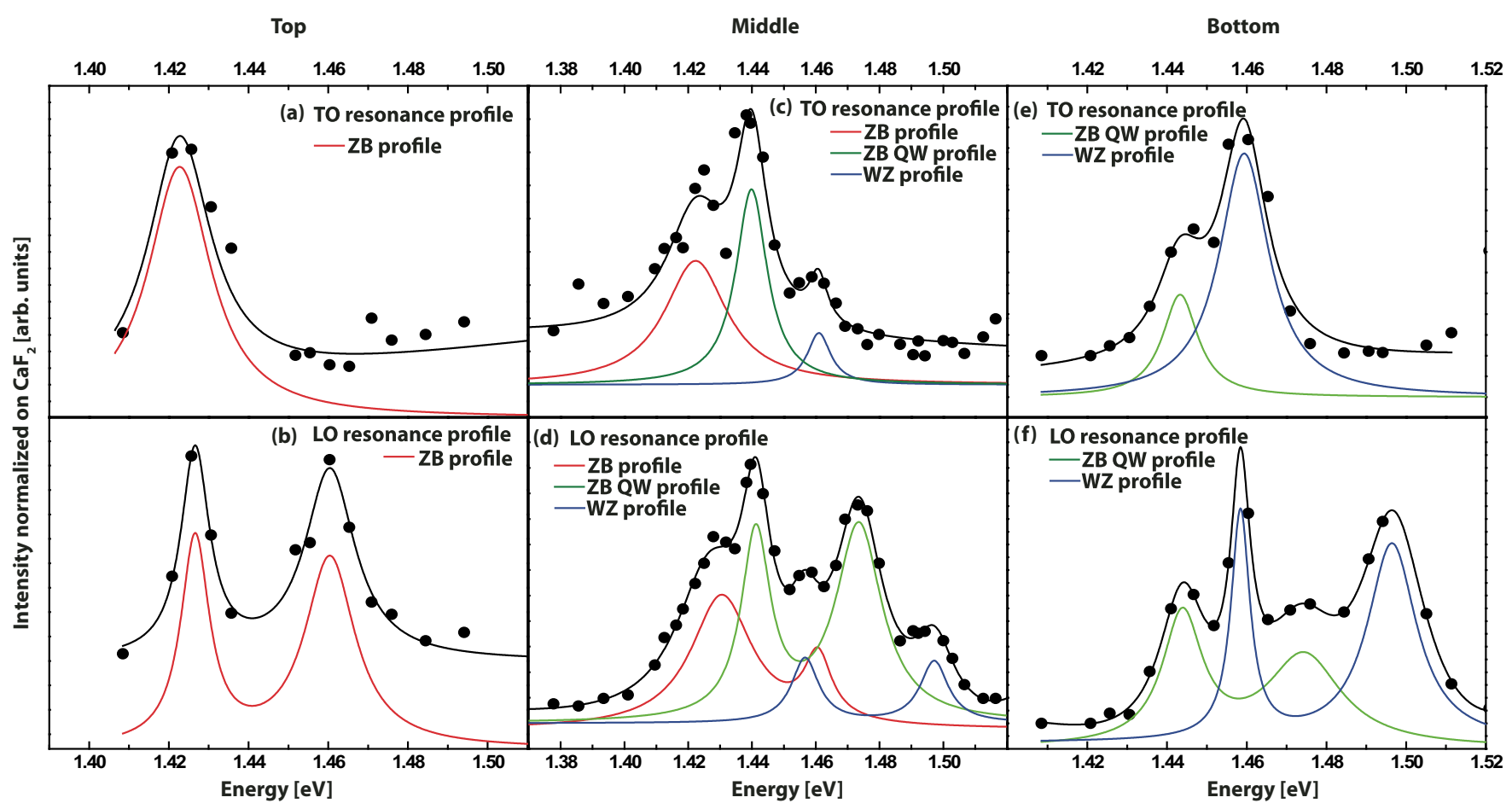

FIG. 3. (Color online) Raman intensity as a function of excitation energy for a single GaAs NW recorded at the top (a)-(b), middle (c)-(d), and bottom (e)-(f) part of the NW. The Raman resonance profiles for scattering by TO (LO) phonons are shown in the upper (lower) panel. The solid lines represent Lorentzian fits to the data with their colors indicating the different origins (ZB: wide zinc-blende segments, WZ: wide wurtzite segments, and ZB QW: thin zinc-blende segments).

two zone-center LO phonons (2LO) leads to a line at $585 \mathrm{~cm}^{-1}$. The ZB and WZ phase cannot be directly distinguished in the Raman spectra, since the separations between the TO and LO phonon frequencies in both phases are too small to be resolved in the present experiments. Furthermore, the observation of the characteristic $E_{2}$ phonon line in WZ GaAs is inhibited by the strong dielectric polarization contrast in the range of photon energies used in the present study [34]. Since there is no difference between the observed TO and LO phonon frequencies and those of bulk GaAs [35], we conclude that the investigated NWs are free of strain.

The PL bands are observed at photon energies between 1.40 and $1.46 \mathrm{eV}$. At the bottom and top of the NW, the PL peak energies lie at $1.46 \mathrm{eV}$ (Fig. 2 bottom) and $1.42 \mathrm{eV}$ (Fig. 2 top), respectively. In these cases very narrow distributions of the PL peak energy are found for excitation at different positions inside the bottom or top region of the NW. In the middle part of the NW, in contrast, the PL peak energy depends strongly on the exact position along the NW axis. In Fig. 2, we show two exemplary spectra from the middle part of the $\mathrm{NW}$ with PL peak energies at 1.40 and $1.44 \mathrm{eV}$, corresponding to the observed range of variation. In accordance with our previous work [31], the PL peak energies at $1.46 \mathrm{eV}$ (bottom region) and $1.42 \mathrm{eV}$ (top region) are attributed to band-to-band recombination in $\mathrm{ZB}$ and $\mathrm{WZ}$ GaAs, respectively. Regarding the bottom region, our assignment is fully consistent with the TEM analysis (cf. Fig. 1) whereas we have to assume the resonant Raman scattering to be dominated by relatively wide $\mathrm{ZB}$ segments in the case of the top region of the particular NW under investigation. The variation of the PL peak energy in a range from below $(1.40 \mathrm{eV})$ to above $(1.44 \mathrm{eV})$ the band gap of $\mathrm{ZB} \mathrm{GaAs}$ at the NW center can be explained by the capture of electrons in relatively narrow ZB segments or SFs and their recombination with holes in adjacent $\mathrm{WZ}$ segments via spatially indirect transitions [17,22], in accordance with the structural information obtained by TEM (cf. Fig. 1) for the middle part of our NWs.

To verify our assignments and to obtain further evidence for the type II band alignment in WZ/ZB heterostructures, we use resonant Raman spectroscopy. The obtained resonance profiles (Raman intensity as a function of the photon energy used for excitation) are shown in Fig. 3 for different positions along the axis of a single NW. The efficiency of Raman scattering is enhanced when the energy of the incident (incoming resonance) or emitted (outgoing resonance) light matches the energy separation of the valence and conduction band corresponding to an optically allowed direct transition. The resonance profiles for scattering by TO phonons [see Figs. 3(a), 3(c), and 3(e)] exhibit only an incoming resonance maximum which is commonly observed for Raman scattering by the deformation-potential mechanism [31,35,36]. The resonance profiles for the LO phonon line [see Figs. 3(b), 3(d), and 3(f)], in contrast, reveal both incoming and outgoing resonance enhancements (separated by one LO-phonon energy of $36 \mathrm{meV}$ ), as expected for Raman scattering induced by the Fröhlich mechanism [12,31,35,36].

The resonance profiles from the top part of the NW [cf. Figs. 3(a) and 3(b)] exhibit incoming and outgoing resonance maxima at $1.425 \pm 0.001 \mathrm{eV}$ (TO and LO) and $1.460 \mathrm{eV}$ (LO), respectively (see Table I). This result can be explained 
TABLE I. Measured Raman resonance energies for TO and LO phonon scattering in wide zinc-blende (ZB) and wurtzite (WZ) segments as well as thin zinc-blende segments (ZB QW) at different positions along the axis of a GaAs NW. Incoming resonances are observed for both TO and LO phonon scattering, outgoing resonances only for LO phonon scattering.

\begin{tabular}{|c|c|c|c|c|c|c|c|}
\hline \multirow{2}{*}{$\begin{array}{l}\text { Position } \\
\text { on wire }\end{array}$} & \multicolumn{3}{|c|}{ Ingoing resonance $(\mathrm{eV})$} & \multicolumn{3}{|c|}{ Outgoing resonance $(\mathrm{eV})$} & \multirow[b]{2}{*}{ Phonon mode } \\
\hline & Zinc-blende & Wurtzite & ZB QW & Zinc-blende & Wurtzite & ZB QW & \\
\hline Top & 1.423 & & & & & & TO \\
\hline Top & 1.426 & & & 1.460 & & & LO \\
\hline Middle & 1.424 & 1.459 & 1.439 & & & & TO \\
\hline Middle & 1.426 & 1.458 & 1.439 & 1.461 & 1.494 & 1.474 & LO \\
\hline Bottom & & 1.459 & 1.443 & & & & TO \\
\hline Bottom & & 1.459 & 1.442 & & 1.495 & 1.476 & LO \\
\hline
\end{tabular}

by Raman scattering dominated by the resonance with the fundamental band gap of $\mathrm{ZB}$ GaAs $\left(E_{\mathrm{ZB}}=1.425 \mathrm{eV}\right)$, in accordance with our above finding from the PL measurements. The profiles from the bottom of the NW [cf. Figs. 3(e) and 3(f)] can be explained by the superposition of resonance enhancements in two different electronic modifications of the NW structure. We assign the stronger component with maxima at $1.459 \mathrm{eV}$ (incoming: TO and LO) and $1.495 \mathrm{eV}$ (outgoing: LO) to the fundamental band gap in wide WZ GaAs segments $\left(E_{\mathrm{WZ}}=1.460 \mathrm{eV}\right)$ [31]. The weaker component exhibits maxima at $1.443 \pm 0.001 \mathrm{eV}$ (incoming: TO and LO) and $1.476 \mathrm{eV}$ (outgoing: LO) and can be explained by Raman scattering in material with an effective band gap between $E_{\mathrm{ZB}}$ and $E_{\mathrm{WZ}}$. In accordance with the PL results discussed above, we assign this second component to narrow $\mathrm{ZB}$ segments. The contribution of this component to the PL spectra from the bottom of the NW (see Fig. 2) might be suppressed because of the preferential capture of carriers in wider WZ GaAs segments with lower band-gap energy. In accordance with the TEM analysis (cf. Fig. 1), the resonance profiles from the center of the NW [cf. Figs. 3(c) and 3(d)] can be explained by a superposition of all components observed at the NWs top and bottom with their characteristic energies given in Table I. Here, the contribution of the thin ZB segments is the strongest one, which might be caused by a stronger resonance enhancement induced by electronic confinement. Most importantly, the effective band gap deduced for thin $\mathrm{ZB}$ segments lies always above the fundamental band gap of $\mathrm{ZB} \mathrm{GaAs}$ as deduced from several additional resonant Raman profiles recorded at different NW positions. This result contrasts our findings from the PL measurements where peak energies below the ZB band gap can be observed (cf. Fig. 2).

To understand the different behavior in PL and Raman experiments observed for the local $\mathrm{ZB} / \mathrm{WZ}$ heterostructure formed by thin $\mathrm{ZB}$ segments in WZ GaAs, we compare the energy ranges of spatially indirect and direct transitions. The corresponding energy diagram is schematically illustrated for a type II band alignment as described in Refs. [17,22]. In this framework SFs are considered to behave electronically like the narrowest possible $\mathrm{ZB}$ segments [17,22]. For a specific ZB segment or a SF, the energy difference between an indirect PL transition and a direct transition is always given by the energy offset in the valence band $(\Delta E)$. The corresponding maximal possible energy ranges for direct and indirect transitions are given by $\left[E_{\mathrm{ZB}}, E_{\mathrm{WZ}}+\Delta E\right]$ and $\left[E_{\mathrm{ZB}}-\Delta E, E_{\mathrm{WZ}}\right]$, respectively. The widths of these energy ranges are determined by the confinement energies in the conduction-band quantum wells (QWs) formed by the ZB segments and SFs (see Fig. 4) with the upper (lower) limit approximating the case of SFs (wide ZB segments). The particular peak positions and widths observed in either kind of experiment reflect essentially the distribution of the ZB segment width inside the specific optical probing area. Consequently, the different energy ranges observed for the PL transitions and the Raman resonance maxima are consistent with the type II band alignment proposed previously $[17,22]$. For the sake of completeness one would have to consider in addition the spontaneous-polarization induced electric fields in the local ZB/WZ heterostructures. The most important impact of such finite electric fields would be a downshift of the energy for indirect transitions [22], but their energy range would still deviate from that of the direct transitions observed by resonant Raman scattering. On the other hand, assuming alternatively a type I band alignment, the barrier heights for $\mathrm{ZB}$ quantum wells would be very shallow, in the range of about 10-30 $\mathrm{meV}\left(E_{\mathrm{WZ}}-E_{\mathrm{ZB}}=35 \mathrm{meV}\right)$ for both the conduction and valence band. In this case only one confined quantum-well state is expected in both bands and the energy ranges for PL transitions and Raman resonance maxima would be essentially the same, even in the presence of spontaneous-polarization induced electric fields. Our experimental observations can thus not be explained by the assumption of a type I band alignment. As a consequence, our combined PL and Raman study provides clear evidence for the type II band alignment in ZB/WZ GaAs heterostructures.

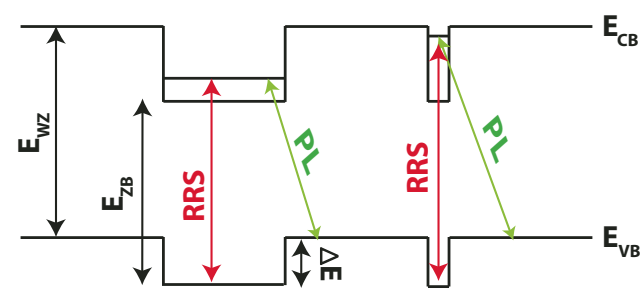

FIG. 4. (Color online) Qualitative model for the band-II alignment for $\mathrm{ZB}$ insertions in a WZ GaAs nanowire. The red arrows show direct band-to-band transitions in a resonant Raman scattering (RRS) process. The green arrows show indirect transitions from the $\mathrm{ZB}$ insertions to the $\mathrm{WZ}$ valance band in a PL process. 
In conclusion, the investigated GaAs NWs have been found to consist of $\mathrm{WZ}$ and $\mathrm{ZB}$ segments with the $\mathrm{WZ}$ phase dominating at the bottom part and the contribution of the $\mathrm{ZB}$ phase increasing towards the top of the NWs. The combination of spatially resolved photoluminescence and resonant Raman spectroscopy has been utilized to provide evidence for the type II band alignment between ZB and WZ GaAs. Furthermore, we confirmed our previous work [31] regarding the band-gap energy of WZ GaAs (35 meV above the band-gap energy of the $\mathrm{ZB}$ phase). All relevant energy values have been obtained from single GaAs NWs using the same experimental conditions.

We acknowledge the ERC under Grant No. 210642 and Deutsche Forschungsgemeinschaft (DFG) under Grant No. Ge2224/2 for financial support.
[1] C. M. Lieber and Z. L. Wang, MRS Bull. 32, 99 (2007).

[2] R. Agarwal and C. M. Lieber, Appl. Phys. A: Mater. Sci. Process 85, 209 (2006).

[3] C. Thelander, P. Agarwal, S. Brongersma, J. Eymery, L. Feiner, A. Forchel, M. Scheffler, W. Riess, B. Ohlsson, and U. Gösele, Mater. Today (Oxford, U.K.) 9, 28 (2006).

[4] V. Mourikr, K. Zuo, S. M. Frolov, S. R. Plissard, E. P. A. M. Bakkers, and L. P. Kouwenhoven, Science 336, 1003 (2012)

[5] M. T. Deng, C. L. Yu, G. Y. Huang, M. Larsson, P. Caroff, and H. Q. Xu, Nano Lett. 12, 6123 (2012).

[6] J. Wallentin, N. Anttu, D. Asoli, M. Huffman, I. Aberg, M. H. Magnusson, G. Siefert, P. F. Keiluweit, B. Ohlsson, F. Dimroth, B. Witzigman, H. Q. Xu, L. Samuelson, K. Deppert, and M. T. Borgström, Science 339, 6123 (2013).

[7] P. Krogstrup, H. I. Jorgensen, M. Heiss, O. Demichel, J. V. Holm, M. Aagsen, J. Nygard, and A. F. i Morral, Nat. Photon. 7, 306 (2013).

[8] K. A. Dick, P. Caroff, J. Bolinsson, M. E. Messing, J. Johansson, K. Deppert, L. R. Wallenberg, and L. Samuelson, Semicond. Sci. Technol. 25, 024009 (2010).

[9] P. Krogstrup, R. Popovitz-Birof, E. Johnson, M. H. Madsen, J. Nygard, and H. Shtrikman, Semicond. Sci. Technol. 10, 4475 (2010).

[10] X. Ren, H. Huang, V. G. Dubrovskii, N. V. Sibirev, M. V. Nazarenko, A. D. Bolshakov, X. Ye, Q. Wang, Y. Huang, X. Zhang, J. Guo, and X. Liu, Sci. Technol. 26, 014034 (2011).

[11] W. Peng, F. Jabeen, B. Jusserand, J. C. Harmand, and M. Bernard, Appl. Phys. Lett. 100, 073102 (2012).

[12] B. Ketterer, M. Heiss, E. Uccelli, J. Arbiol, and A. F. i Morral, ACS Nano 5, 7585 (2011).

[13] M. Heiss, S. Conesa-Boj, J. Ren, H.-H. Tseng, A. Gali, A. Rudolph, E. Uccelli, F. Peiró, J. R. Morante, D. Schuh, E. Reiger, E. Kaxiras, J. Arbiol, and A. Fontcuberta i Morral1, Phys. Rev. B 83, 045303 (2011).

[14] M. Moewe, L. C. Chuang, S. Crankshaw, C. Chase, and C. Chang-Hasnain, Appl. Phys. Lett. 93, 023116 (2008).

[15] A. De and C. E. Pryor, Phys. Rev. B 81, 155210 (2010).

[16] Z. Zanolli, F. Fuchs, J. Furthmüller, U. von Barth, and F. Bechstedt, Phys. Rev. B 75, 245121 (2007).

[17] D. Spirkoska, J. Arbiol, A. Gustafsson, S. Conesa-Boj, F. Glas, I. Zardo, M. Heigoldt, M. H. Gass, A. L. Bleloch, S. Estrade, M. Kaniber, J. Rossler, F. Peiro, J. R. Morante, G. Abstreiter, L. Samuelson, and A. Fontcuberta i Morral, Phys. Rev. B 80, 245325 (2009).
[18] T. B. Hoang, A. F. Moses, H. L. Zhou, D. L. Dheeraj, B. O. Fimland, and H. Weman, Appl. Phys. Lett. 94, 133105 (2009).

[19] F. Martelli, M. Piccin, G. Bais, F. Jabeen, S. Ambrosini, S. Rubini, and A. Franciosi, Nanotechnology 18, 125603 (2007).

[20] M. Murayama and T. Nakayama, Phys. Rev. B 49, 4710 (1994).

[21] R. Gurwitz, A. Tavor, L. Karpeles, I. Shalish, W. Yi, G. Seryogin, and V. Narayanamurti, Appl. Phys. Lett. 100, 191602 (2012).

[22] U. Jahn, J. Lähnemann, C. Pfüller, O. Brandt, S. Breuer, B. Jenichen, M. Ramsteiner, L. Geelhaar, and H. Riechert, Phys. Rev. B 85, 045323 (2012)

[23] L. Ahtapodov, J. Todorovic, P. Olk, T. Mjland, P. Slttnes, D. L. Dheeraj, A. T. J. van Helvoort, B.-O. Fimland, and H. Weman, Nano Lett. 12, 6090 (2012).

[24] B. V. Novikov, S. Y. Serov, N. G. Filosofov, I. V. Shtrom, V. G. Talalaev, O. F. Vyvenko, E. V. Ubyivovk, Y. B. Samsonenko, A. D. Bouravleuv, I. P. Soshnikov, N. V. Sibirev, G. E. Cirlin, and V. G. Dobrovskii, Phys. Status Solidi RRL 4, 175 (2010).

[25] A. M. Graham, P. Corfdir, M. Heiss, S. Conesa-Boj, E. Uccelli, A. Fontcuberta i Morral, and R. T. Phillips, Phys. Rev. B 87, 125304 (2013).

[26] D. C. Kim, D. L. Dheeraj, B. O. Fimland, and H. Weman, Appl. Phys. Lett. 102, 142107 (2013).

[27] J. Bao, D. C. Bell, F. Capasso, J. B. Wagner, T. Mårtensson, J. Trägårdh, and L. Samuelson, Nano Lett. 8, 836 (2008).

[28] P. Corfdir, B. Van Hattem, E. Uccelli, A. Fontcuberta i Morral, and T Phillips, Appl. Phys. Lett. 103, 133109 (2013).

[29] M. Cardona and G. Güntherodt, Light Scattering in Solids II (Springer, Berlin, 1982).

[30] S. Breuer, M. Hilse, A. Trampert, L. Geelhaar, and H. Riechert, Phys. Rev. B 82, 075406 (2010).

[31] P. Kusch, S. Breuer, M. Ramsteiner, L. Geelhaar, H. Riechert, and S. Reich, Phys. Rev. B 86, 075317 (2012).

[32] P. Tronc, Y. E. Kitaev, G. Wang, M. F. Limonov, A. G. Panfilov, and G. Neu, Phys. Status Solidi B 216, 599 (1999).

[33] J. L. Birman, Phys. Rev. 114, 1490 (1959).

[34] M. Ramsteiner, O. Brandt, P. Kusch, S. Breuer, S. Reich, and L. Geelhaar, Appl. Phys. Lett. 103, 043121 (2013).

[35] P. Yu and M. Cardona, Fundamentals of Semiconductors (Springer, Berlin, 2005).

[36] M. Brewster, O. Schimek, S. Reich, and S. Gradecak, Phys. Rev. B 80, 201314 (2009). 